\title{
Mutational optimization of the coelenterazine-dependent luciferase from Renilla
}

\author{
Jongchan Woo ${ }^{1,2}$ and Albrecht G von Arnim*1
}

Address: ${ }^{1}$ Department of Biochemistry, Cellular and Molecular Biology, The University of Tennessee, Knoxville, TN 37996-0840, USA and ${ }^{2}$ The Rockefeller University, Molecular, Cell and Developmental Biology, 1230 York Avenue New York, NY 10065, USA

Email: Jongchan Woo - jwoo@mail.rockefeller.edu; Albrecht G von Arnim* - vonarnim@utk.edu

* Corresponding author

Published: 30 September 2008

Plant Methods 2008, 4:23 doi:10.1/86/1746-48|I-4-23

This article is available from: http://www.plantmethods.com/content/4/1/23

(C) 2008 Woo and von Arnim; licensee BioMed Central Ltd.

This is an Open Access article distributed under the terms of the Creative Commons Attribution License (http://creativecommons.org/licenses/by/2.0), which permits unrestricted use, distribution, and reproduction in any medium, provided the original work is properly cited.
Received: 9 August 2008

Accepted: 30 September 2008

\begin{abstract}
Renilla luciferase (RLUC) is a popular reporter enzyme for gene expression and biosensor applications, but it is an unstable enzyme whose catalytic mechanism remains to be elucidated. We titrated that one RLUC molecule can turn over about one hundred molecules of coelenterazine substrate. Mutagenesis of active site residue Pro220 extended the half-life of photon emission, yielding brighter luminescence in $E$. coli. Random mutagenesis uncovered two new mutations that stabilized and increased photon emission in vivo and in vitro, while ameliorating substrate inhibition. Further amended with a previously identified mutation, a new triple mutant showed a threefold improved $\mathrm{k}_{\mathrm{cat}}$, as well as elevated luminescence in Arabidopsis. This advances the utility of RLUC as a reporter protein, biosensor, or resonance energy donor.
\end{abstract}

\section{Background}

Renilla luciferase (RLUC) is a cofactor-less, single subunit, blue light emitting luciferase isolated from the marine anthozoan Renilla reniformis (RLUC, E.C. number 1.13.12.5, luciferin-2-monooxygenase, decarboxylating) [1]. Aside from its utility as a reporter for gene expression assays, RLUC has also found application in assays for protein interaction based on fragment complementation [2] and bioluminescence resonance energy transfer [3]. The substrate of Renilla luciferase, coelenterazine, consists of a central aromatic imidazopyrazinone, which is derivatized with p-hydroxy-phenyl (R1), benzyl (R2), and phydroxy-benzyl (R3) moieties. Using molecular oxygen, RLUC catalyzes an oxidative decarboxylation in which the imidazole ring is opened and carbon dioxide is released [4-7]. Relaxation of the electronically excited coelenteramide reaction product is accompanied by emission of a photon of blue ( $470 \mathrm{~nm})$ light.
Compared to the calcium-stimulated photoprotein, aequorin, and its relatives, which utilize the same substrate as RLUC, the catalytic mechanism of RLUC is not yet well understood $[8,9]$. Photoproteins are single turnover enzymes. Removal of the coelenteramide product and binding of a fresh substrate molecule require a reducing agent and the concomitant removal of calcium [10]. RLUC is not homologous to aequorin but evolved from an $\alpha / \beta$ hydrolase ancestor closely related to current bacterial dehalogenases. Its structure has recently been solved [11]. Within the large hydrophobic active site, the putative catalytic triad consists of Asp120, His285, and Glu144. Mutagenesis data and inactivation with diethylpyrocarbamate indicate that His285 is important for catalysis, presumably as a general base $[12,13]$. A model for how coelenterazine and its peroxidized reaction intermediate might be positioned in the active site has been proposed [13]. 
Re-engineering of the RLUC sequence might ameliorate undesirable properties that arise upon expression in heterologous hosts, which lack RLUCs two partner proteins, a green fluorescent protein and a calcium-responsive coelenterazine binding protein $[14,15]$. Previous consensusguided mutagenesis has already led to RLUC versions with improved stability in serum, improved ability to utilize the purple-emitting substrate, bisdeoxycoelenterazine, and altered spectral properties $[12,16]$. RLUC is well known to be inactivated in the presence of substrate, resulting in most of the light to be emitted as a flash of a few seconds in length. While a short half-life of the enzyme might be beneficial for time-resolved gene expression studies, it is undesirable for protein-interaction studies based on bioluminescence resonance energy transfer $[5,17,18]$.

Here, we describe the results of site directed and random mutagenesis in conjunction with expression and purification of recombinant RLUC enzyme in E. coli with the goal of improving specific enzymatic parameters of RLUC. We describe novel mutants with increased $\mathrm{k}_{\mathrm{cat}^{\prime}}$ extended halflife of photon emission in vitro and in vivo, and enhanced light emission upon expression in plant cells.

\section{Methods \\ Mutagenesis and other recombinant DNA techniques}

The wild type Renilla reniformis luciferase cDNA obtained from plasmid pBS-35S-RLUC-attR (Genbank accession, AY995136) [17] was subcloned into the expression vector pET30(a) as an NcoI-BamHI fragment, thus adding an Nterminal histidine tag and linker sequence (His-RLUC) [13]. For random mutagenesis, the RLUC CDNA was amplified using an error-prone PCR procedure, GeneMorpho ${ }^{\circledast}$ II Random Mutagenesis (Stratagene, La Jolla, CA). A library of 1300 putative mutant clones (strain BL21(DE3)) was grown in LB in white 96-well microtiter plates (Packard, Meriden, CT) to an optical density of about 0.6. Colonies were surveyed for RLUC activity in the presence of $2 \mu \mathrm{M}$ native coelenterazine (Biotium, Hayward, CA) in the PolarStar plate reader (BMG Labtech, Durham, NC). Candidates with elevated RLUC activity were reconfirmed by inducing RLUC expression at OD = 0.5 with $1 \mathrm{mM} \mathrm{IPTG}$ for 1 hour at $30^{\circ} \mathrm{C}$ and the mutation was identified by DNA sequencing. Subsequently, mutations were also introduced into a human codon-optimized RLUC cDNA (hRLUC, Genbank accession, AAK53368, Packard, Meriden, CT). Site directed mutagenesis was performed using the Quickchange procedure (Stratagene, La Jolla, CA). Mutations were confirmed by resequencing of the entire RLUC coding region to guard against unintended secondary mutations. To generate recombination cloning vectors, the appropriate fragments of pBS-35S-hRLUC-attR (Genbank accession AY995138) and pBS-35S-attR-hRLUC (Genbank accession
AY995140) [17] were replaced with the corresponding NheI/BglII restriction fragment from pET30(a)-SuperhRLUC that contained the M185V, K189V, and V267I mutations. The entire SuperhRLUC coding regions of the recombination vectors were confirmed by sequencing.

\section{Expression and purification of RLUC}

RLUC expression in E. coli strain BL21(DE3)pLysS was induced with $1 \mathrm{mM}$ IPTG for $3 \mathrm{~h}$ hours at $30^{\circ} \mathrm{C}$. The accumulation of RLUC in E. coli was routinely checked by cell lysis and gel electrophoresis and Coomassie Blue-staining. Mutant proteins generally accumulated to similar levels. RLUC was purified from the soluble cytosolic fraction over a nickel column (His-Bind Kit, Novagen, Darmstadt, Germany) following standard procedures that included sonication, centrifugation of cell debris at $12,000 \mathrm{rpm}$ for 10 minutes at $4{ }^{\circ} \mathrm{C}$, and filtration of the supernatant through a 0.45 micron filter to prevent clogging of resin. Protein was affinity purified according to the manufacturer's protocol and eluted with $1 \mathrm{M}$ imidazole, $0.5 \mathrm{M}$ $\mathrm{NaCl}, 20 \mathrm{mM}$ Tris-HCl, pH, 7.9. After elution, RLUC was dialyzed overnight against $2 \mathrm{~L}$ of phosphate buffered saline (PBS, pH7.2) in order to remove imidazole. Protein concentration was determined using the BCA assay (Pierce, Rockford, IL) with BSA as a standard. Alternatively, the protein concentration of preparations that were free of imidazole was measured by UV-absorbance using an extinction coefficient of $65,040 \mathrm{M}^{-1} \mathrm{~cm}^{-1}$ [19]. Purified RLUC protein was stored in PBS with 50\% glycerol at $70^{\circ} \mathrm{C}$ in small aliquots or stored at $4^{\circ} \mathrm{C}$ for up to 2 weeks.

\section{Kinetics of RLUC enzyme activity}

Enzyme assays were conducted using freshly purified RLUC enzyme at a concentration of $1 \mathrm{nM}$ or as otherwise indicated in $1 \mathrm{ml}$ PBS ( $\mathrm{pH}$ 7.2). Native coelenterazine substrate was added from a $250 \times$ stock solution of the indicated concentration in ethanol (final ethanol concentration, $0.8 \%$ ), the solution was mixed by tapping to ensure a maximal supply of oxygen, and the luminescence activity was recorded in the TD20/20 luminometer (Turnerdesigns, Sunnyvale, CA). The first 5-second luminescence reading was taken as a measure of enzyme activity. The $K_{M}$ values of wild type RLUC and selected mutants were calculated according to standard Michaelis-Menten theory using Prism software (GraphPad Software Inc., San Diego, CA) from at least 3 repeat measurements. Several independent protein preparations yielded similar $K_{M}$ values.

\section{Emission spectra}

Luminescence spectra were recorded under the same condition as the enzyme assay using a spectroluminometer (Photon Technology International, Inc., Birmingham, $\mathrm{NJ})$, except that the assay volume was $2 \mathrm{ml}$. Native coelenterazine substrate was $2 \mu \mathrm{M}$ (ethanol concentration, 
$0.8 \%$ ). Protein concentration was $10 \mathrm{nM}$ purified enzyme or as otherwise indicated. Generally, the emission spectrum was analyzed with the Felix32 software (Photon Technology International, Inc., Birmingham, NJ). All spectra were recorded at $1 \mathrm{~nm}$ per second from short to long wavelength. No adjustments for detector sensitivity or luminescence decay over time were made; nevertheless, the spectra are directly comparable among each other and emphasize the differences in emission in the short-wavelength region.

\section{Substrate/enzyme titration}

The amount of RLUC enzyme needed to deplete a nearly saturating amount of substrate $(0.1 \mathrm{ml}$ of $1 \mu \mathrm{M}$ or $10 \mu \mathrm{M}$ coelenterazine) was determined by titration. Parallel reactions were set up with RLUC at concentrations between $100 \mathrm{nM}$ and $1 \mathrm{nM}$ in $100 \mu \mathrm{l}$ of assay buffer $(50 \mathrm{mM}$ potassium phosphate, pH 7.4, $500 \mathrm{mM} \mathrm{NaCl}, 1$ mM EDTA) [5]. Reactions were allowed to proceed for at least 2 hours until luminescence had decayed to near-background levels. Each spent reaction was then split into two aliquots. To one aliquot fresh enzyme $(10 \mathrm{nM})$ was added. If no increase in luminescence was observed it was concluded that the substrate must have been used up completely allowing us to deduce the stoichiometry between enzyme and substrate. The second $50 \mu \mathrm{l}$ aliquot was supplemented with $10 \mu \mathrm{M}$ substrate to check whether the substrate had been used up completely by the enzyme or whether RLUC activity had been depleted.

\section{Spectrophotometric assay of RLUC activity}

The spectrum of a $10 \mu \mathrm{M}$ solution of coelenterazine in assay buffer was recorded. RLUC was added to $100 \mathrm{nM}$ and the spectrum re-recorded after 10 minutes and 60 minutes of reaction time. The extent of spontaneous degradation of coelenterazine was determined in a control reaction without added enzyme.

\section{Plant growth condition and transgenic lines}

Columbia wild type and transgenic seedlings were germinated on $0.8 \%$ agar medium containing Murashige and Skoog salts (MS; Sigma, St. Louis) and 1\% sucrose without antibiotics. The transgenic plants expressing the hRLUC or SuperhRLUC cassettes were grown on a MS selection media containing $1.5 \mathrm{mg} / \mathrm{l}$ ammonium glufosinate herbicide (Basta; Sigma, St. Louis).

\section{Measurement of Renilla luciferase activity in transgenic Arabidopsis}

Luminescence units were measured from 10 day-old seedlings in the presence of $2 \mu \mathrm{M}$ coelenterazine (Biotium, Hayward, CA) in water using a TD-20/20 tube luminometer that is equipped with the blue-color filter. After adding $2 \mu \mathrm{M}$ coelenterazine, samples were incubated in darkness for $10 \mathrm{~min}$ at room temperature to allow the substrate to penetrate into plants and to allow delayed chlorophyll autofluorescence to decay [17].

\section{Protein extraction from plants and western blotting}

Plant protein was extracted with passive lysis buffer from the Dual-Luciferase ${ }^{\circledR}$ Reporter Assay System (Promega, Madison, WI) and RLUC detected by western blotting using a monoclonal antibody (Chemicon, Temecula, CA).

\section{Photon-counting images}

Transgenic 7-10 days old Arabidopsis seedlings were imaged on a Nikon microscope with a Hamamatsu C2400 ICCD (Meyer Instruments, Houston, TX). Seedlings were pre-incubated in $2 \mu \mathrm{M}$ coelenterazine for $5 \mathrm{~min}$ and then photon emission was recorded for $5 \mathrm{~min}$. Images were pseudo-colored with ImageJ (NIH, Bethesda, MD).

\section{Results and discussion}

Previous docking simulations and mutagenesis of RLUC active site residues D120, E144, and H285, which are conserved with the catalytic triad of the dehalogenase LinB, suggested that they also underlie the catalytic mechanism of RLUC. Meanwhile, two other residues, N53 and W121, were proposed to function in binding to the R1 ring of coelenterazine [13]. When the neighboring P220 was mutated to ten different residues, several mutants displayed the unusual characteristic that E. coli in vivo luminescence was initially low, but rose dramatically over time, soon surpassing the peak luminescence of cells expressing wild-type RLUC (Figure 1A) [13]. When tested in vitro after purification, the P220G and P220L mutant proteins showed only $4 \%$ and $16 \%$ of the initial luciferase activity of wild-type RLUC, respectively (not shown). However, compared to wild-type RLUC and many other mutants tested, whose half-life of photon emission in vitro was about 40 seconds, P220G and P220L yielded a strikingly more stable luminescence output over time (Figure 1B).

It is unclear why RLUC rapidly loses its enzymatic activity when in contact with substrate. The turnover number of RLUC is low (111 $\mu \mathrm{mol} \mathrm{min}^{-1} \mu \mathrm{mol}^{-1}$ enzyme) [5] but the sum total of substrate molecules turned over by one molecule of RLUC has not been reported. The loss of luminescence activity cannot be attributed to oxygen depletion because the concentration of dissolved oxygen in water $\left(280 \mu \mathrm{M}\right.$ at $\left.20^{\circ} \mathrm{C}\right)$ is much higher than that needed to react with coelenterazine $(1 \mu \mathrm{M})$. By titrating the amount of wild-type enzyme needed to deplete the luminescence potential of a solution of coelenterazine substrate, we determined that one molecule of enzyme is able to turn over up to 100 molecules of native coelenterazine (Figure $2 \mathrm{~A})$. To confirm that a sufficient amount of RLUC causes complete turnover of the substrate, the enzymatic reaction was also followed by measuring absorbance of coelenter- 
A
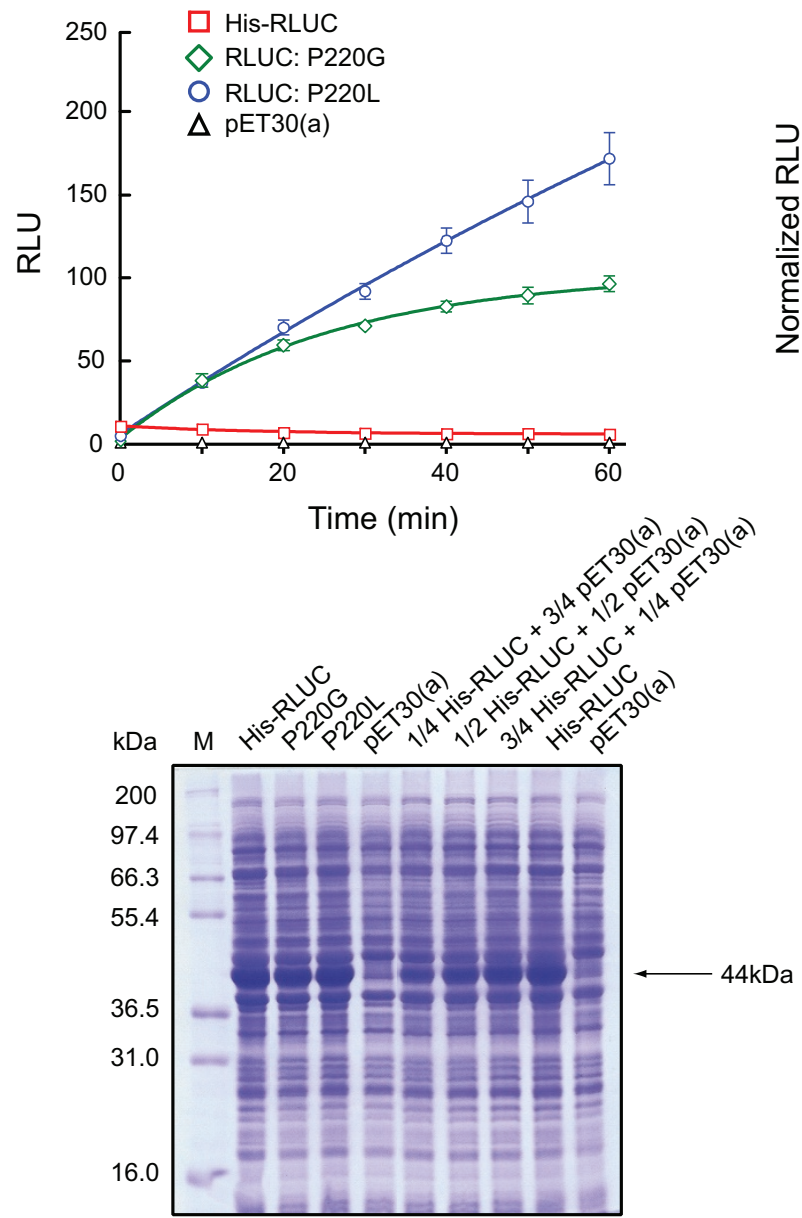

B
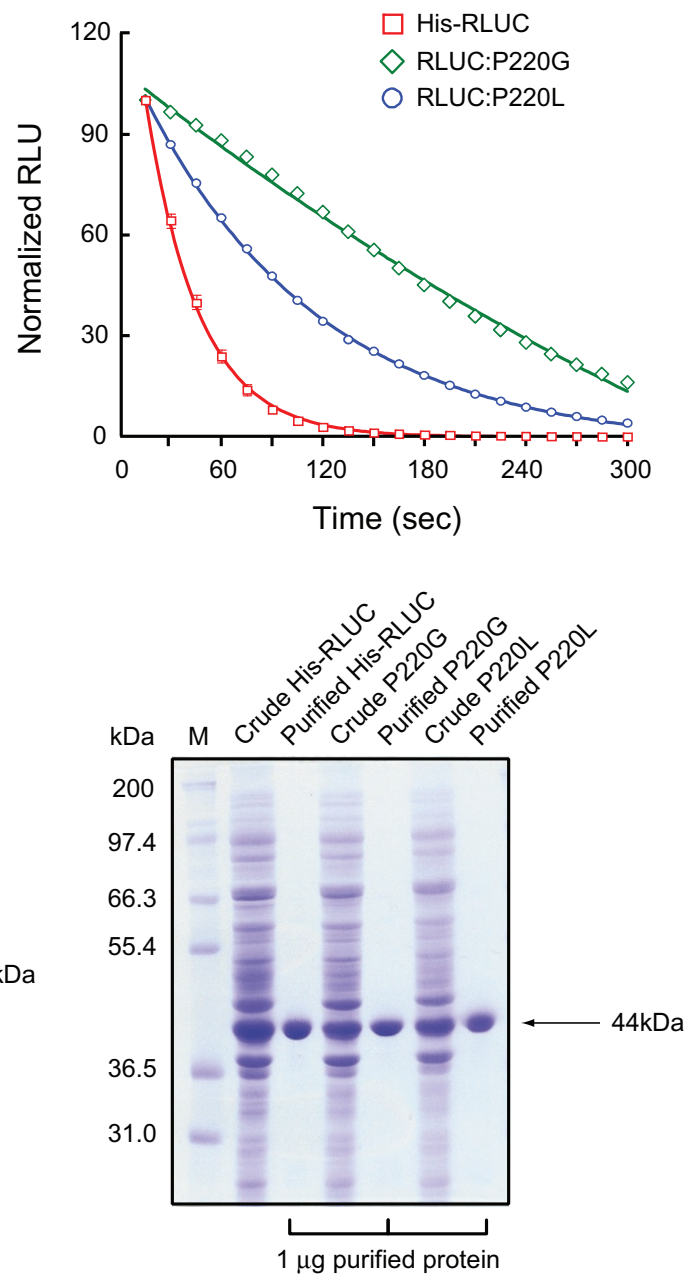

Figure I

Half-life of RLUC enzyme activity. (A) Time course of in vivo luminescence in E. coli cells expressing wild type RLUC and two P220 mutants. (B) Time course of in vitro luminescence of wild type RLUC and two P220 mutants. Enzyme and substrate concentrations were $10 \mathrm{nM}$ and $2 \mu \mathrm{M}$ respectively in PBS buffer $\mathrm{pH}$ 7.2. Absolute activities were normalized for better comparison (first time-point $=100$ ). Results shown are averages from three biological replicates each performed in triplicate. The Coomassie stained SDS-PAGE gels below show that the P220 mutants and wild-type RLUC accumulate to equivalent levels in E. coli. RLU stands for relative luminescence units.

azine at $420 \mathrm{~nm}$ (Figure 2B). The vast majority of photons are emitted within the first ten minutes of the reaction (Figure 1B), which coincides with the drop in coelenterazine absorbance at $420 \mathrm{~nm}$ and appearance of a new peak at $350 \mathrm{~nm}$, which is attributed to coelenteramide. Therefore, the drop in luminescence cannot simply be explained by a drop in quantum efficiency. Coelenteramide is a strong competitive inhibitor of RLUC $\left(\mathrm{K}_{\mathrm{I}} \sim 23\right.$ nM) [6], and its accumulation must contribute to the loss of activity. However, it cannot be solely responsible, because enzyme activity is also lost at enzyme concentrations as low as $1 \mathrm{pM}$, when, in light of the titration data, 
A

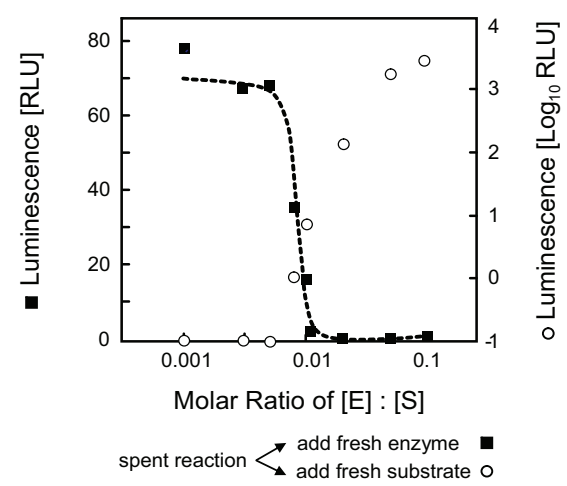

B

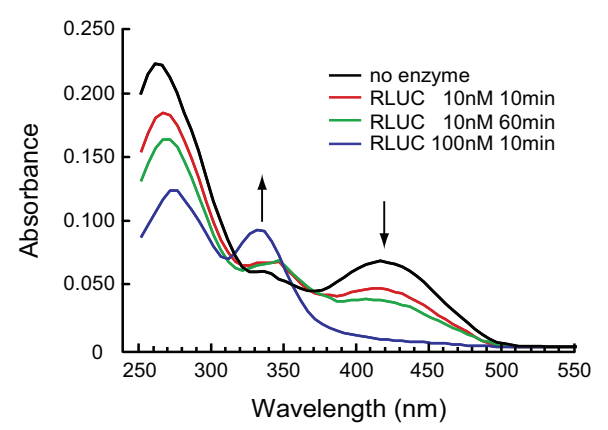

C

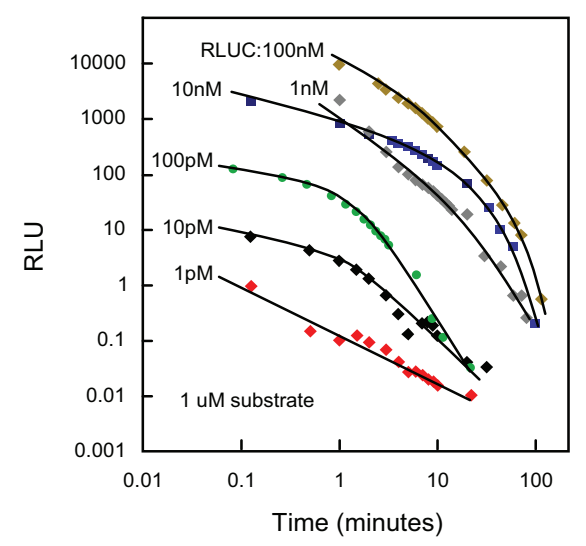

Figure 2

Decay properties of RLUC enzyme activity. (A) A titration assay indicates that I molecule of RLUC can turn over $\sim 100$ molecules of coelenterazine. A I $\mu$ M solution of native coelenterazine substrate was incubated with increasing amounts of RLUC enzyme ( $\mathrm{nM}$ to $100 \mathrm{nM}$ ) and the reaction was allowed to go to completion. Once light emission had ceased, the reaction was split in half and supplemented with fresh enzyme (squares) to examine whether all substrate had been depleted; or with fresh substrate (circles) to check for residual enzyme activity. Note, if an excess of substrate was added, the loss of RLUC activity was not reversible by adding fresh substrate. (B) Spectrophotometric confirmation of substrate turnover by RLUC. Absorbance spectra were recorded for $10 \mu \mathrm{M}$ coelenterazine before (black trace) and after (blue trace) turnover by $100 \mathrm{nM}$ RLUC. Two traces of partially completed reactions are shown for comparison (red and green traces). (C) Half life of RLUC activity in the in vitro assay as measured by luminescence decay kinetics. 
the concentration of accumulated coelenteramide is far below the $\mathrm{K}_{\mathrm{I}}$ (Figure 2C).

Next, we screened RLUC mutants generated by random mutagenesis for mutants with increased peak of light emission in E. coli or increased stability of light emission over time. Upon DNA sequencing and protein purification, the activities of two mutants, V267I and K189V, were elevated over wild-type RLUC (Table 1). A third mutant, M185G, was of interest because of a slightly increased half life (not shown), although $\mathrm{k}_{\mathrm{cat}}$ was decreased. While K189 and M185 lie in the presumptive gateway that guides the substrate into the active site [11], V267 lies outside of the active site, as do most of the eight mutations constituting the RLUC8 mutant, which has an increased $\mathrm{k}_{\text {cat }}[12]$.

The coelenteramide reaction product relaxes from the excited state to the ground state under emission of a photon of light. The precise peak wavelength of light emission is influenced by the protonation state of the reaction product and is also dependent on the physical environment (hydrophobicity) in the active site [20]. The emission spectra of K189V and M185G were similar to wildtype RLUC (not shown). In contrast, V267I showed two major peaks, a blue-shifted shoulder of $390 \mathrm{~nm}$, indicative of the formation of a neutral coelenteramide [7,2123] and a blue-shifted maximum at $450 \mathrm{~nm}$ [see Additional file 1].

The putative beneficial mutations, K189V and V267I, were combined into a double mutant, named RLUC+. The two mutations appeared to act additively, yielding a more

Table I: Activities of RLUC mutants selected for improved enzymatic activity.

\begin{tabular}{|c|c|c|c|}
\hline Mutations & Name & Activity $\pm S D$ & Condition \\
\hline \multicolumn{4}{|l|}{ Native RLUC cDNA } \\
\hline none & His-RLUC & 100 & in vitro I) \\
\hline V267I & - & $163 \pm 33$ & in vitro \\
\hline $\mathrm{K} 189 \mathrm{~V}$ & - & $128 \pm 16$ & in vitro \\
\hline $\mathrm{K}|89 \mathrm{~V}+\mathrm{V} 267|$ & RLUC+ & $317 \pm 82$ & in vitro \\
\hline$M 185 V+K 189 V+V 267 I$ & SuperRLUC & $411 \pm 113$ & in vitro \\
\hline \multicolumn{4}{|c|}{ Codon-optimized RLUC cDNA (hRLUC) } \\
\hline none & His-hRLUC & 100 & in vivo 2 ) \\
\hline $\mathrm{KI} / 89 \mathrm{~V}$ & - & $175 \pm 70$ & in vivo \\
\hline$M 185 V+K 189 \mathrm{~V}$ & - & $425 \pm 120$ & in vivo \\
\hline$M 185 V+K I 89 V+V 267 I$ & SuperhRLUC & $475 \pm 130$ & in vivo \\
\hline
\end{tabular}

I) In vitro activites are initial luminescence values upon addition of substrate ( $2 \mu \mathrm{M}$ coelenterazine). The unmutated version was set to 100.

2) In vivo activities are luminescence activities from $E$. coli strain BL2I without IPTG induction ( $2 \mu \mathrm{M}$ coelenterazine). than two-fold increase in the apparent $\mathrm{k}_{\mathrm{cat}}$ of light emission compared to wild-type RLUC (Figure 3A). From another set of RLUC mutations that yielded increased luminescence in mammalian cells $[12,24]$, we selected M185V, a mutation that increased the stability of enzyme activity in serum and the ability to luminesce using the substrate bis-deoxycoelenterazine [12]. Its incorporation into RLUC+ yielded a slight further increase in light emission (SuperRLUC; Figure 3A). Including the P220G mutation provided no further enhancement (not shown). Neither RLUC+ nor SuperRLUC had an altered $\mathrm{K}_{\mathrm{M}}$ and their emission spectra were similar to wild type (Figure 3A and $3 \mathrm{~B}$ ). Interestingly, the $\mathrm{K} 189 \mathrm{~V}$ mutation suppressed the blue shift and the marked purple emission detected in the V267I mutant. However, the luminescence of SuperRLUC had a two-fold longer half-life in vitro compared to wild-type RLUC (Figure 3C). RLUC is known to be inhibited by aggregation at high concentrations of substrate (above $3 \mu \mathrm{M}$ ) [6]. RLUC+ and SuperRLUC were less sensitive to substrate inhibition (Figure 3D). Elevated RLUC activity was also observed when the mutations were introduced into a codon-optimized cDNA (hRLUC, Table 1).

SuperhRLUC gave rise to approximately two-fold higher luminescence values than regular hRLUC when expressed in stably transgenic Arabidopsis seedlings (Figure 4A and 4B). In addition, SuperhRLUC protein extracted from plants had a prolonged half life of photon emission [see Additional file 2]. For easy construction of SuperhRLUC fusion proteins, new recombination cloning vectors harboring SuperhRLUC were generated (Figure 4C); these are available from the corresponding author upon request. The improved luciferase activity and stability of SuperhRLUC in Arabidopsis should be beneficial for utilization of RLUC as a reporter protein or biosensor. Why RLUC suffers from irreversible inactivation of its enzymatic activity still requires further study. Dramatic improvements in luminescence emission can be expected if this limitation can be resolved.

\section{Conclusion}

Mutant versions of Renilla luciferase with increased $\mathrm{k}_{\mathrm{cat}}$ were identified from a library of random mutations expressed in E. coli. A combination of two or three mutations resulted in increased activity of the Renilla luciferase reporter enzyme in transgenic Arabidopsis.

\section{Abbreviations \\ RLU: relative light units; RLUC: Renilla luciferase.}

\section{Competing interests}

The authors declare that they have no competing interests. All authors read and approved the final manuscript. 


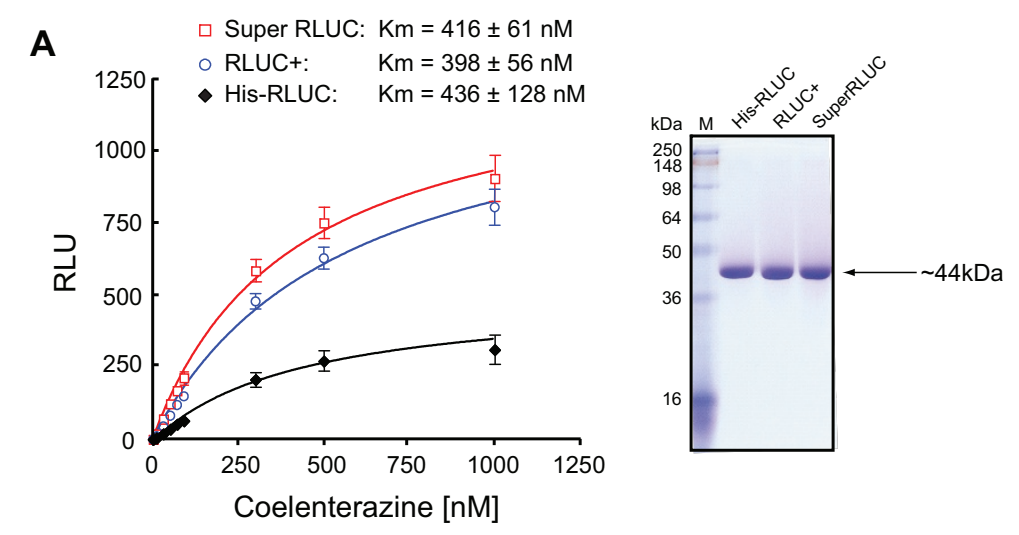

B

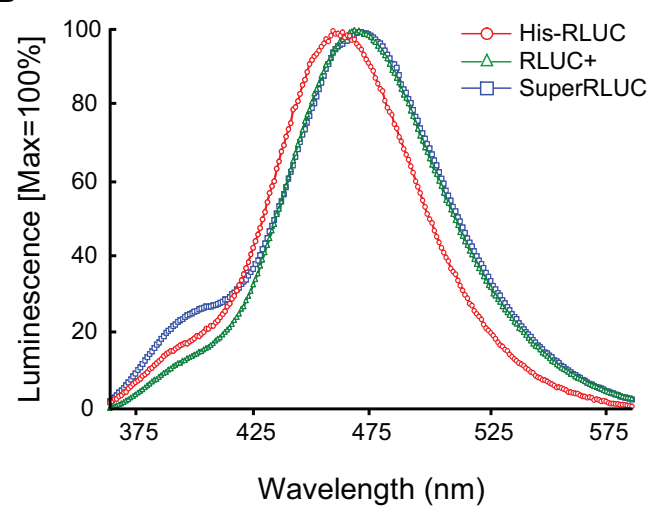

D
C

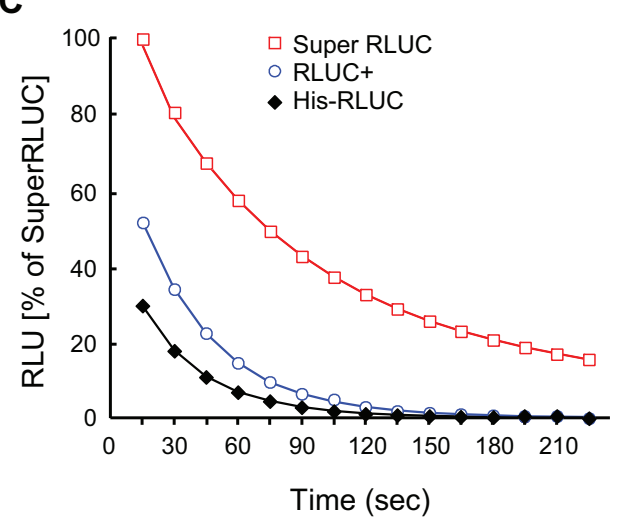

Figure 3

Enzyme kinetics of optimized RLUC proteins. (A) Derivation of $K_{M}$ values. The $K_{M}$ for wild-type RLUC was similar to previously published data such as $210 \mathrm{nM}$ (coelenterazine $h)^{5}$ and $300 \mathrm{nM}^{23}$. Also shown are wild-type RLUC, RLUC+ and SuperRLUC, which were purified by nickel affinity chromatography and run on a polyacrylamide gel. (B) Luminescence spectra for RLUC, RLUC+ and Super-RLUC. (C) Time course of luminescence activity. Note the increased half life of activity of the RLUC+ and SuperRLUC mutants. (D) Inhibition of RLUC activity by high substrate concentration. 

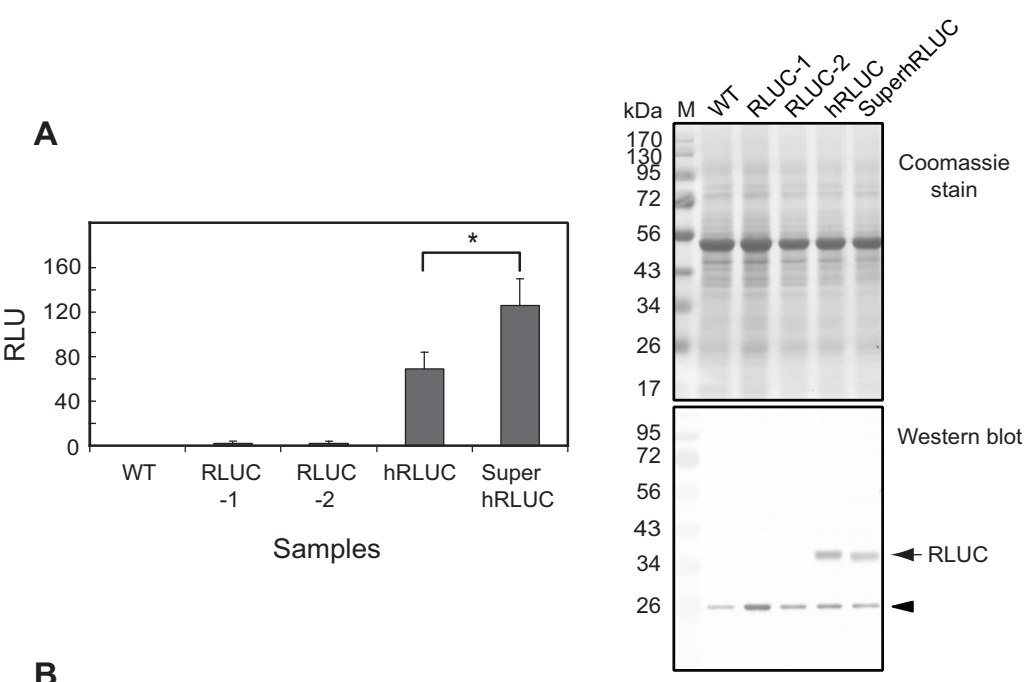

B
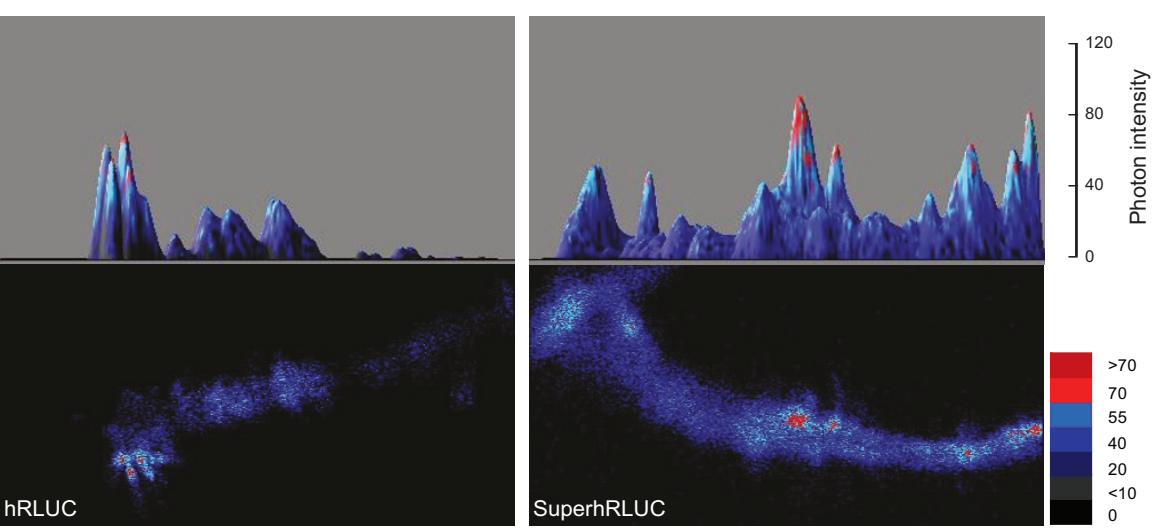

C

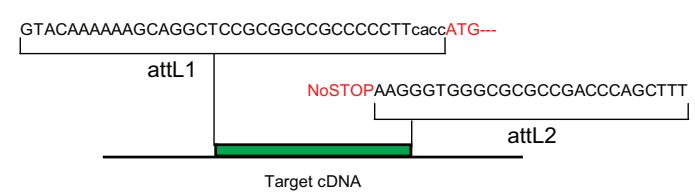

pENTR clones

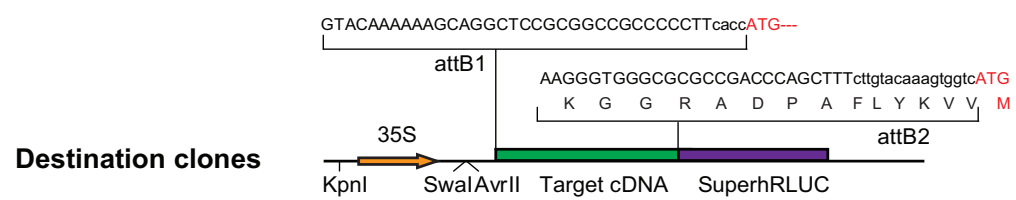

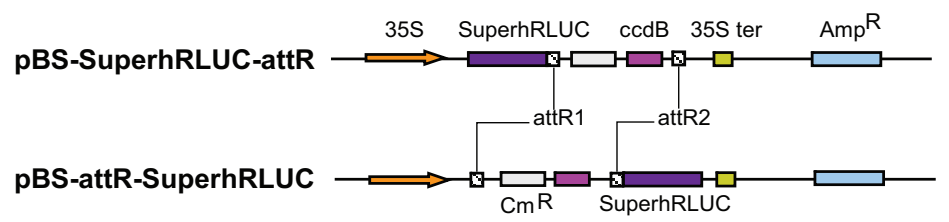

Figure 4 (see legend on next page) 
Figure 4 (see previous page)

Improvement of light emission by SuperhRLUC in Arabidopsis and recombination vectors. (A) In vivo luminescence measurement of transgenic Arabidopsis expressing regular RLUC (2 lines), hRLUC, or SuperhRLUC. Asterisk represents the significant increase of luciferase activity of SuperhRLUC over hRLUC $(P<0.01$, two tailed t-test; $n=4$ repeats with 25 seedlings each). The immunoblot below probed with RLUC antibody confirms that hRLUC and SuperhRLUC (36 kDa) accumulate to similar, high, levels; the original RLUC can only be detected on the immunoblot after prolonged development. The arrowhead indicates a non-specific immunoreaction. (B) Photon-counting images of representative seedling roots. Photon emission in the primary roots of SuperhRLUC transgenic Arabidopsis was stronger compared to regular hRLUC (lower panels). The upper panels show a 3-dimensional version of the images below in which photon intensity is encoded in the third axis. Seedlings were incubated in $2 \mu \mathrm{M}$ coelenterazine and imaged for $5 \mathrm{~min}$. (C) pBS-SuperhRLUC-attR and pBS-attR-SuperhRLUC are recombination vectors for expression of SuperhRLUC fusion proteins, which contain the lambda att recombination sites utilized by the Gateway ${ }^{\mathrm{TM}}$ (Invitrogen) system [25]. Sequence elements flanking an insert (target CDNA) are shown before $(p E N T R)$ and after (Destination) attL $\times$ attR recombination. The $35 \mathrm{~S}$ indicates a strong promoter in plants, which can be replaced by restriction digestion with Kpnl and Swal or Avrll. The ccdB gene provides for counter-selection of non-recombinants.

\section{Authors' contributions}

JW and AGV designed experiments and wrote the manuscript. JW performed all experiments except those in Figure 2, which were performed by AGV.

\section{Additional material}

\section{Additional file 1}

Luminescence spectra. Luminescence emission scans of selected single mutants with improved enzymatic properties.

Click here for file

[http://www.biomedcentral.com/content/supplementary/17464811-4-23-S1.pdf]

\section{Additional file 2}

Enzyme stability measurements. Enzyme stabilities of RLUC, hRLUC, and SuperhRLUC after extraction from transgenic Arabidopsis $(10 \mu \mathrm{M}$ coelenterazine; $n=4$; average \pm s.d.).

Click here for file

[http://www.biomedcentral.com/content/supplementary/17464811-4-23-S2.pdf]

\section{Acknowledgements}

This work was supported by National Science Foundation grants MCB0I 14653 and DBI-0619631. We are grateful for enzymological advice from Liz Howell and Dan Roberts and comments on the manuscript by ByungHoon Kim. We thank Tim Sparer for access to the spectroluminometer and the Center for Environmental Biotechnology for access to the photon counting camera.

\section{References}

I. Lorenz WW, McCann RO, Longiaru M, Cormier MJ: Isolation and expression of a cDNA encoding Renilla reniformis luciferase. Proc Natl Acad Sci USA I991, 88:4438-4442.

2. Paulmurugan R, Gambhir SS: Monitoring protein-protein interactions using split synthetic Renilla luciferase protein-fragment-assisted complementation. Anal Chem 2003, 75:1584- 1589 .

3. $\mathrm{Xu} Y$, Piston DW, Johnson $\mathrm{CH}$ : A bioluminescence resonance energy transfer (BRET) system: application to interacting circadian clock proteins. Proc Natl Acad Sci USA I999, 96: I 5 I- I 56.
4. Hori K, Wampler JE, Matthews JC, Cormier MJ: Identification of the product excited states during the chemiluminescent and bioluminescent oxidation of Renilla (sea pansy) luciferin and certain of its analogs. Biochemistry 1973, I 2:4463-4468.

5. Matthews JC, Hori K, Cormier MJ: Purification and properties of Renilla reniformis luciferase. Biochemistry 1977, I 6:85-91.

6. Matthews JC, Hori K, Cormier MJ: Substrate and substrate analogue binding properties of Renilla luciferase. Biochemistry 1977, 16:5217-5220.

7. Ohmiya Y, Hirano T: Shining the light: the mechanism of the bioluminescence reaction of calcium-binding photoproteins. Chem Biol 1996, 3:337-347.

8. Head JF, Inouye S, Teranishi K, Shimomura O: The crystal structure of the photoprotein aequorin at $2.3 \AA$ resolution. Nature 2000, 405:372-376.

9. Liu ZJ, Stepanyuk GA, Vysotski ES, Lee J, Markova SV, Malikova NP, Wang BC: Crystal structure of obelin after Ca2+-triggered bioluminescence suggests neutral coelenteramide as the primary excited state. Proc Natl Acad Sci USA 2006, 103:2570-2575.

10. Shimomura O, Johnson FH: Regeneration of the photoprotein aequorin. Nature 1975, 256:236-238.

II. Loening AM, Fenn TD, Gambhir SS: Crystal structures of the luciferase and green fluorescent protein from Renilla reniformis. J Mol Biol 2007, 374:1017-1028.

12. Loening AM, Fenn TD, Wu AM, Gambhir SS: Consensus guided mutagenesis of Renilla luciferase yields enhanced stability and light output. Protein Eng Des Sel 2006, I 9:391-400.

13. Woo JC, Howell MH, von Arnim AG: Structure-function studies on the active site of the coelenterazine-dependent luciferase from Renilla. Protein Sci 2008, I 7:725-735.

14. Ward WW, Cormier MJ: An energy transfer protein in coelenterate bioluminescence. Characterization of the Renilla green-fluorescent protein. J Biol Chem 1979, 254:78I-788.

15. Titushin MS, Markova SV, Frank LA, Malikova NP, Stepanyuk GA, Lee J, Vysotski ES: Coelenterazine-binding protein of Renilla muelleri: cDNA cloning overexpression and characterization as a substrate of luciferase. Photochem Photobiol Sci 2008, 7: I89-196.

16. Loening AM, Wu AM, Gambhir SS: Red-shifted Renilla reniformis luciferase variants for imaging in living subjects. Nat Methods 2007, 4:64I-643.

17. Subramanian C, Woo J, Cai X, Xu X, Servick S, Johnson $\mathrm{CH}$, Nebenführ A, von Arnim AG: A suite of tools and application notes for in vivo protein interaction assays using bioluminescence resonance energy transfer (BRET). Plant J 2006, 48: I38-I52.

18. Xu X, Soutto M, Xie Q, Servick S, Subramanian C, von Arnim AG, Johnson $\mathrm{CH}$ : Imaging protein interactions with bioluminescence resonance energy transfer (BRET) in plant and mammalian cells and tissues. Proc Natl Acad Sci USA 2007, 104: |0264-10269.

19. Mach H, Middaugh CR, Lewis RV: Statistical determination of the average values of the extinction coefficients of tryptophan and tyrosine in native proteins. Anal Biochem 1992, 200:74-80. 
20. Stepanyuk GA, Golz S, Markova SV, Frank LA, Lee J, Vysotski ES: Interchange of aequorin and obelin bioluminescence color is determined by substitution of one active site residue of each photoprotein. FEBS Lett 2005, 579:1008-I0|4.

21. Hart RC, Matthews JC, Hori K, Cormier MJ: Renilla reniformis bioluminescence: luciferase-catalyzed production of nonradiating excited states from luciferin analogues and elucidation of the excited state species involved in energy transfer to Renilla green fluorescent protein. Biochemistry 1979, 18:2204-2210.

22. Shimomura O: Cause of spectral variation in the luminescence of semisynthetic aequorins. Biochem J 1995, 306:537-543.

23. Vysotski ES, Lee J: $\mathbf{C a}^{2+}$-regulated photoproteins: structural insight into the bioluminescence mechanism. Acc Chem Res 2004, 37:405-415.

24. Hoshino $H$, Nakajima $Y$, Ohmiya $Y$ : Luciferase-YFP fusion tag with enhanced emission for single-cell luminescence imaging. Nat Methods 2007, 4:637-639.

25. Landy A: Dynamic structural and regulatory aspects of lambda site-specific recombination. Annu Rev Biochem 1989, 58:9|3-949.

Publish with Bio Med Central and every scientist can read your work free of charge

"BioMed Central will be the most significant development for disseminating the results of biomedical research in our lifetime. "

Sir Paul Nurse, Cancer Research UK

Your research papers will be:

- available free of charge to the entire biomedical community

- peer reviewed and published immediately upon acceptance

- cited in PubMed and archived on PubMed Central

- yours - you keep the copyright 